\title{
Heterochronic blood exchange attenuates age-related neuroinflammation and confers cognitive benefits: do microvascular protective effects play a role?
}

\author{
Derek M. Huffman • Anna Csiszar • Zoltan Ungvari
}

Received: 6 January 2021 / Accepted: 19 January 2021 / Published online: 28 January 2021

(C) American Aging Association 2021

Parabiosis, a technique dating back to the midnineteenth century, has re-emerged over the past 15 years as a widely used experimental tool to enable unique insights into cell-autonomous and cell nonautonomous contributors to aging [1-4]. The ability to demonstrate that either an aged phenotype can be transposed to a young animal or rejuvenation can be conferred to an old organ or cell by the young environment provides a fascinating, critical proof-of-concept that external factors may be involved in such effects. Importantly, a proper and careful interpretation of the clues provided by such studies can guide the next steps toward possible mechanistic insights. Indeed, there are now several examples of purported pro- and anti-geronic factors identified from parabiosis and plasma transfer

D. M. Huffman $(\bowtie)$

Department of Molecular Pharmacology and Medicine, The Institute for Aging Research, Albert Einstein College of Medicine, 1300 Morris Park Avenue, Forchheimer Bldg., Rm. 236, Bronx, NY 10461, USA

e-mail: derek.huffman@einsteinmed.org

A. Csiszar $\cdot$ Z. Ungvari

Vascular Cognitive Impairment and Neurodegeneration Program, Reynolds Oklahoma Center on Aging/Center for Geroscience and Healthy Brain Aging, Department of Biochemistry and Molecular Biology, University of Oklahoma Health Sciences Center, Oklahoma City, OK, USA

\section{Z. Ungvari}

International Training Program in Geroscience, Doctoral School of Basic and Translational Medicine/Department of Public Health, Semmelweis University, Budapest, Hungary studies that have been the focus of intense study and proposed to serve as either targets for replacement or removal as a means to treat specific manifestations of aging.

However, as with most any experimental paradigm, heterochronic parabiosis and exchange consists of caveats and limitations that must be appreciated when interpreting results. One such scenario arises in instances where apparent rejuvenation is observed, as suggested by Mehdipour et al. [5] and prior work from the Conboy laboratory [6]. On one hand, such effects could be elicited by an anti-geronic factor(s) passing to the older animal to restore youthful function, but the old environment also may unintentionally benefit in this setting from the dilution of potential bad actors, and in the case of parabiosis, the sharing of young organs during this chronic exchange. Indeed, this is what is demonstrated here by Mehdipour et al. [5] whereby a single neutral blood exchange (NBE) in old mice improved multiple facets of brain health, including cognitive function, neuroinflammation and markers of senescence [5], which is consistent with prior reports from the Conboy lab [6]. Interestingly, the effect of blood dilution may be even more fascinatingly interesting and complex than previously appreciated, in that the apparent relenting of pro-geronic factors via dilution acted to "reset" a plethora of circulating factors in the old environment, which could in turn mediate at least some of the beneficial effects in this model. Such is the case when Mehdipour et al. identify elevations in circulating VEGF, BDNF, and TIMP2, among others, all of which are implicated in brain health, just days after performing 
NBE or therapeutic plasma exchange (TPE). Such an observation is highly relevant to a better understanding of data generated via the heterochronic parabiosis and blood exchange paradigms, in that evidence of rejuvenation should not only consider the possibility of antigeronic or pro-geronic factors as direct mediators per se, but also factor in the complexity of "resetting" the secretome, both systemically, as well as the niche in which cells of interest reside. However, these results do not discount the importance of blood-borne factors in aging, but rather further support the important, albeit complex role that cell non-autonomous mechanisms play in our understanding of aging.

Although this study by the Conboy laboratory reveals new insights into NBE-induced rejuvenation, several questions remain to be answered. For one, the role of cerebromicrovascular mechanisms contributing to decreased neuroinflammation and rescue of cognitive dysfunction needs to be elucidated. The blood-brain barrier partitions the brain from circulating blood and functions to potentially shield neuronal cells as well as microglia from changes in circulating blood-borne pro-geronic and anti-geronic factors. Cerebromicrovascular endothelial cells are major constituents of the blood-brain barrier and are the only cell type in the brain that come into direct contact with all the circulating factors whose levels are altered by heterochronic blood exchange. Importantly, recent studies demonstrate that heterochronic parabiosis exerts significant rejuvenating effects on endothelial cells in the peripheral circulation [7] and further implicate a role of cerebromicrovascular endothelial cells in the mediation of these effects via candidate anti-geronic [8] and pro-geronic circulating factors [9], respectively.

Mehdipour et al. also demonstrated that old mice after a single NBE exhibit reduced neuroinflammation, characterized by decreased microglia activation. Importantly, previous studies demonstrate that an age-related disruption of the blood-brain barrier promotes neuroinflammation, which plays a causal role in the pathogenesis of neurodegeneration and cognitive decline $[10,11]$. Specifically, through the damaged blood-brain barrier, plasma constituents (including IgG, thrombin, and fibrinogen) can enter the brain parenchyma, which promotes neuroinflammation by activating microglia [12]. Thus, these intriguing results provoke many additional questions, such as: How does NBE affect cerebromicrovascular endothelial cell phenotypes and BBB integrity? What are the roles of the endothelial protective effects of NBE in reduced neuroinflammation? In addition, the possibility that NBEinduced endothelial protection also plays an important role in improved cerebral blood flow needs to be explored. There is strong evidence that rescue of cerebromicrovascular endothelial function and restoration of endothelium-mediated neurovascular coupling responses in the aged mouse brain confers significant cognitive benefits [13]. Importantly, heterochronic parabiosis was shown to restore endothelium-mediated vasorelaxation [7] and improve brain capillarization [2] in aged mice. Embracing these new insights should further enable the field to reach an undiluted truth toward the important role that systemic factors play in the aging process.

\section{References}

1. Conboy MJ, Conboy IM, Rando TA. Heterochronic parabiosis: historical perspective and methodological considerations for studies of aging and longevity. Aging Cell. 2013;12(3):525-30.

2. Villeda SA, Luo J, Mosher KI, Zou B, Britschgi M, Bieri G, et al. The ageing systemic milieu negatively regulates neurogenesis and cognitive function. Nature. 2011;477(7362):90-4.

3. Conboy IM, Conboy MJ, Wagers AJ, Girma ER, Weissman IL, Rando TA. Rejuvenation of aged progenitor cells by exposure to a young systemic environment. Nature. 2005;433(7027):760-4.

4. Yousefzadeh MJ, Wilkinson JE, Hughes B, Gadela N, Ladiges WC, Vo N, et al. Heterochronic parabiosis regulates the extent of cellular senescence in multiple tissues. Geroscience. 2020;42(3):951-61.

5. Mehdipour M. Mehdipour T. Wong N, Liu C, Chen CC, et al. Plasma dilution improves cognition and attenuates neuroinflammation in old mice. Geroscience: Skinner CM; 2020.

6. Mehdipour M, Skinner C, Wong N, Lieb M, Liu C, Etienne $\mathrm{J}$, et al. Rejuvenation of three germ layers tissues by exchanging old blood plasma with saline-albumin. Aging (Albany NY). 2020;12(10):8790-819.

7. Kiss T, Tarantini S, Csipo T, Balasubramanian P, Nyul-Toth A, Yabluchanskiy A, et al. Circulating anti-geronic factors from heterochonic parabionts promote vascular rejuvenation in aged mice: transcriptional footprint of mitochondrial protection, attenuation of oxidative stress, and rescue of endothelial function by young blood. Geroscience. 2020;42(2): $727-48$. 
8. Ozek C, Krolewski RC, Buchanan SM, Rubin LL. Growth differentiation factor 11 treatment leads to neuronal and vascular improvements in the hippocampus of aged mice. Sci Rep. 2018;8(1):17293.

9. Yousef H, Czupalla CJ, Lee D, Chen MB, Burke AN, Zera $\mathrm{KA}$, et al. Aged blood impairs hippocampal neural precursor activity and activates microglia via brain endothelial cell VCAM1. Nat Med. 2019;25(6):988-1000.

10. Winkler EA, Bell RD, Zlokovic BV. Pericyte-specific expression of PDGF beta receptor in mouse models with normal and deficient PDGF beta receptor signaling. Mol Neurodegener. 2010;5:32.

11. Montagne A, Barnes SR, Sweeney MD, Halliday MR, Sagare AP, Zhao Z, et al. Blood-brain barrier breakdown in the aging human hippocampus. Neuron. 2015;85(2):296302 .
12. Davalos D, Ryu JK, Merlini M, Baeten KM, Le Moan N, Petersen MA, et al. Fibrinogen-induced perivascular microglial clustering is required for the development of axonal damage in neuroinflammation. Nat Commun. 2012;3:1227.

13. Tarantini S, Valcarcel-Ares MN, Toth P, Yabluchanskiy A, Tucsek Z, Kiss T, et al. Nicotinamide mononucleotide (NMN) supplementation rescues cerebromicrovascular endothelial function and neurovascular coupling responses and improves cognitive function in aged mice. Redox Biol. 2019;24:101192.

Publisher's note Springer Nature remains neutral with regard to jurisdictional claims in published maps and institutional affiliations. 